\section{Pesquisa em, sobre e para os serviços de saúde: panorama internacional e questões para a pesquisa em saúde no Brasil}

\author{
Research in, about, and for health services: \\ an international overview and questions \\ for health research in Brazil
}

Hillegonda Maria Dutilh Novaes 1

Os serviços de saúde são hoje estruturas organizacionais e técnicas extremamente diversifi-

${ }^{1}$ Faculdade de Medicina, Universidade de São Paulo, São Paulo, Brasil.

Correspondência H. M. D. Novaes

Departamento de Medicina Preventiva, Faculdade de Medicina, Universidade de São Paulo. Av. Dr. Arnaldo 455, 2 o andar, São Paulo, SP 01246-903, Brasil. hidutilh@usp.br

\begin{abstract}
Health systems and services are important determinants of health conditions and quality of life. Health services research is a multidisciplinary area aimed at deepening and widening knowledge on the area in specific contexts and focusing on system accessibility, impact, and performance and conditions and quality of health care in order to contribute to decisions by policy-makers, managers, and health professionals. This article analyzes the main characteristics and trends in recent output in the international literature and discusses several key questions for health services research in Brazil.
\end{abstract}

Health Services; Health Services Research; Health Care Quality, Access, and Evaluation cadas, incluindo desde consultórios individuais e unidades básicas até hospitais terciários e especializados, bem como serviços de apoio diagnóstico e terapêutico. A condição essencial para sua identificação é ser o espaço onde se localizam os profissionais e as tecnologias materiais responsáveis pela realização da atenção à saúde da população.

Os serviços se transformam continuamente no que diz respeito ao perfil tanto dos profissionais, com uma ampliação nas categorias inseridas nos processos assistenciais, com especialização horizontal (tipos de profissionais) e vertical (tipos de especialistas nas categorias profissionais), quanto das tecnologias materiais incorporadas e dos procedimentos diagnósticos e terapêuticos realizados. Isso faz com que os serviços de saúde, atualmente sempre integrados a "sistemas de atenção à saúde", constituam-se em objetos que desafiam categorizações e dificultam as análises que buscam identificar e mensurar a sua participação e o seu impacto sobre os problemas de saúde de populações especificadas.

Uma questão inicial que se coloca é a própria definição dos seus limites e da sua inserção, seja no espaço físico das cidades, seja na dinâmica de vida da população a ser atendida. O distanciamento, tanto físico como metafísico, entre o domicílio e os serviços de saúde, o 
lugar do viver individual e familiar e as organizações de prestação de serviços com características particulares, tem sido problematizado nas análises sobre acesso e acessibilidade ao sistema e serviços de saúde. Constata-se sempre que as suas "portas de entrada" não se abrem com facilidade e de forma diferenciada para demandantes diversos 1 .

No entanto, propostas que buscam melhorar essa acessibilidade pelo esmaecimento das fronteiras que os separam, como os programas de saúde da família, ou de home care, deparam com dificuldades inesperadas na definição das responsabilidades dos envolvidos na atenção e na definição e realização das intervenções técnicas. Com o desenvolvimento dos hospitais modernos, no século XIX, e a expansão dos serviços e sistemas de saúde desde então, a prática médica, que se inseria fundamentalmente na esfera privada da vida social, deslocou-se para a esfera pública. A reaproximação da prática médica com a esfera privada nos tempos atuais não se mostra simples, nem poderia se dar com facilidade 2 .

Atualmente, é nos serviços de saúde que acontece a quase totalidade dos procedimentos diagnósticos e terapêuticos, bem como a atenção à saúde individual, sob a responsabilidade dos profissionais de saúde (com tudo o que isso significa modernamente), com participação significativa na determinação das condições de vida e saúde/doença das populações. Todavia, os serviços de saúde são, com alguma freqüência, inadequadamente reconhecidos pelo seu papel específico nos estudos sobre os fatores de risco associados à morbi-mortalidade de indivíduos e populações, ou de eficácia e efetividade de intervenções em pacientes, nas pesquisas desenvolvidas pela clínica e pela epidemiologia. Isto é, não são identificados os efeitos das características dos serviços sobre os pacientes, as quais interferem nas práticas tecnológicas individuais, ao serem organizações que articulam de forma complexa dimensões tecnológicas com aquelas gerenciais, políticas, econômicas e culturais.

A atenção à saúde é considerada, nas sociedades contemporâneas ocidentais, um direito fundamental do cidadão, tendo adquirido uma dimensão econômica crescente pela extensão da sua cobertura populacional e pela constituição de um importante "complexo médico-industrial”. Isso estimula o desenvolvimento da temática relativa à saúde em várias áreas de conhecimento, incluindo a Gestão e Administração, as Ciências Políticas e Sociais, Economia e Ciências Humanas em geral. Observa-se, contudo, que nos estudos dessas áreas os serviços de saúde são pouco problematizados nas suas dimensões tecnológicas específicas.

Desde os anos 50, nos países desenvolvidos, foi reconhecida a necessidade da realização de estudos que tivessem como objeto específico os serviços de saúde, tanto na perspectiva "interna" - conhecer os processos de atenção neles desenvolvidos e seus efeitos sobre a saúde e a doença dos pacientes atendidos -, como na "externa" - conhecer o seu desempenho e participação na conformação dos sistemas de saúde e seu impacto sobre a saúde da população. Na qualidade de prática científica, essa temática esteve inserida, ainda que em escala relativamente reduzida até os anos 80 , nas atividades desenvolvidas nesse período por diferentes grupos de pesquisadores e com referenciais teóricos e políticos variados. Fez parte dos intensos debates sobre os determinantes dos processos de saúde/doença, medicina e sociedade, políticas de saúde, desenvolvimento científico e tecnológico, epidemiologia social e epidemiologia clínica.

Em 1992, a Organização Pan-Americana da Saúde (OPAS) publicou uma antologia sobre a investigação em serviços de saúde 3 , em que é possível acompanhar, no que diz respeito à produção vinculada à clínica e epidemiologia, a dinâmica desse campo desde os anos 50, considerado pelos seus organizadores como um campo multidisciplinar de pesquisa, e não uma nova especialidade com várias disciplinas. É interessante observar que autores importantes no desenvolvimento da epidemiologia nos anos 50 e 60, como Morris e Cochrane, tiveram os serviços de saúde como objeto de interesse, o primeiro na perspectiva dos resultados nos pacientes e o segundo na da eqüidade em saúde 4,5 . Em 1968, criam-se nos Estados Unidos, três anos após a implantação de seus programas Medicare e Medicaid, propostas de atenção pública à saúde para grupos populacionais de risco social e sanitário (idosos e pobres), o National Institute of Health Services Research, que passa a financiar parte importante das pesquisas clínicas, epidemiológicas e organizacionais, que tomam os serviços de saúde como campo para investigação, sendo também objeto desta 6 .

As décadas de 70 e 80 foram marcadas por uma expansão quase explosiva tanto da cobertura populacional pelos sistemas de saúde, isto é, a proporção da população com acesso a algum tipo de assistência como parte da implantação de políticas sociais e de saúde, quanto da sua densidade tecnológica, ou seja, dos tipos de tecnologias de produto e processo incorporados e utilizados nos serviços de saúde. Essa expansão se deu de forma mais intensa nos países de- 
senvolvidos do que nos "em desenvolvimento" e, se, de um lado, é considerada como essencialmente positiva pelos governos, pelas indústrias e pela população, a sua intensidade gera muito rapidamente preocupações quanto à sua sustentabilidade econômico-financeira e política, bem como efetividade técnica e impacto na saúde populacional. Isso contribui para o desenvolvimento de áreas diversas de avaliação em saúde (tecnológica, econômica, de programas, da qualidade em serviços com maior destaque) e de gestão e planejamento em saúde 7,8,9,10.

No final da década de 80 , também como parte das preocupações com os custos e resultados da atenção à saúde, fortalece-se o discurso de que, além da produção de novos conhecimentos sobre os serviços e sistemas de saúde e seu desempenho, faz-se necessária uma maior integração entre o conhecimento clínico, epidemiológico e de planejamento e gestão, com sua articulação em sínteses que sejam capazes de orientar as ações a serem desenvolvidas. $\mathrm{Ou}$ seja, devem ser desenvolvidas competências na adequada tradução e transferência do conhecimento científico e tecnológico, além de inovações em políticas - no sentido macro e, principalmente, micro, de policies - e práticas capazes de obter resultados e impactos mais positivos, reduzindo os negativos 11,12. Sendo assim, em 1989, nos Estados Unidos, sustentada por grandes expectativas políticas, é criada a Agency for Health Care and Policy Research (AHCPR) (que, no início dos anos 2000, passa a ser Agency for Healthcare Research and Quality-AHRQ). Responsável por financiar pesquisas e propostas de intervenção nessa direção, é bastante indicativa da visibilidade que esses movimentos passam a adquirir.

A década de 90, em termos muito esquemáticos, caracterizou-se pelo enfraquecimento da concepção dos Estados nacionais como principais responsáveis pelo bem-estar da sua população, por crises importantes nas políticas sociais e de saúde, pela globalização da economia, aumento das desigualdades sociais e nacionais e transformações nos modelos adotados como referência para as análises das relações entre ciência-tecnologia-inovação e desenvolvimento social e econômico 13,14,15. De um lado, coloca-se a visão de uma Ciência toda poderosa, a macrociência, consubstanciada em bombas atômicas, armamentos cada vez mais destruidores e grandes projetos na área de energia e transportes, que passam a inspirar uma certa ciência e tecnofobia (não influenciando em absoluto, no entanto, o perfil dos investimentos nos países que dominam essas áreas). De outro, evidencia-se o papel da microciência (consubstanciada na expressão "sociedade baseada no conhecimento"), presente na valorização da educação, no desenvolvimento de processos de decisão baseados no uso racional de conhecimentos e na idéia de que o cientificismo da vida política em geral poderá fortalecer o poder das sociedades na superação das crises e levar ao bem-estar social 16,17,18.

Nesse contexto, desenvolveram-se, na década de 90, diferentes iniciativas na área da saúde que se propuseram atuar sobre os diversos pontos que medeiam os caminhos possíveis entre o conhecimento e a saúde, ao reconhecer que as articulações entre eles não se dão natural ou automaticamente. Produziram-se conhecimentos e tecnologias com o objetivo de contribuir para melhores decisões políticas, econômicas, de gestão e técnicas, necessárias para a produção de impactos positivos, em todos os níveis, com um certo privilégio para a atuação nos sistemas e serviços de saúde 11 .

Uma categorização possível dessas iniciativas pode ser feita pela dimensão na qual priorizam a sua atuação: as tecnologias de produto (pesquisa e desenvolvimento de medicamentos, imunobiológicos e equipamentos considerados essenciais para a saúde) ou de processo (sistemas, serviços e profissionais de saúde). Essas últimas, que constituem o grupo de interesse para a discussão aqui proposta, diferenciam-se, novamente, de acordo com o objeto privilegiado, os sistemas de saúde, ou os serviços e profissionais de saúde. As iniciativas que se inserem nesses dois grupos apresentam características comuns e se distinguem principalmente pelos atores que delas participam, pelas alianças políticas estabelecidas e tecnologias de intervenção privilegiadas.

Nas iniciativas que têm os sistemas de saúde como seu objeto de interesse identificam-se como atores importantes desde organizações internacionais oficiais (Organização Mundial da Saúde - OMS -, OPAS) e não governamentais diversas (Council on Health Research and Development, Alliance for Health Policy and Systems Research), até organizações públicas nacionais, principalmente em países desenvolvidos (como, por exemplo, o Centre for Review and Dissemination do Serviço Nacional de Saúde britânico). Essas iniciativas buscam melhorar o seu desempenho, destacando entre seus objetivos prioritários (além da efetividade e eficiência) a necessidade de maior inclusão e eqüidade na assistência.

Um outro conjunto de iniciativas procura contribuir para a melhor formação e atuação dos profissionais, por meio da facilitação do acesso à informação científica, como os pro- 
gramas de bibliotecas eletrônicas e a produção de informação mais diretamente aplicável na prática cotidiana, como os guidelines ou diretrizes clínicas, produzidos por associações de especialistas ou organizações governamentais e não governamentais 19 . Por sua vez, as iniciativas que procuram atuar sobre os serviços têm como objetivos mais freqüentes contribuir para sua maior efetividade técnica, eficiência econômica, sua qualidade e a dos programas de atenção à saúde, incluindo-se aí propostas diversas de "acreditação", desenvolvimento de bases de dados com sínteses de avaliações tecnológicas e econômicas, sistemas de monitoramento apoiados em indicadores e instrumentos diversos (como, por exemplo, os reportcards para os hospitais nos Estados Unidos) 20.

É nesse panorama que se insere o crescimento nos países desenvolvidos (mais intenso nos Estados Unidos e Canadá), nos anos 90, de pesquisas e publicações que se consideram como sendo health services research, ou pesquisa em serviços de saúde, refletindo também um relativo maior apoio por parte de agências financiadoras de pesquisas $21,22,23$. São pesquisas com características muito diversificadas, que têm em comum dois aspectos básicos: os serviços de saúde como objeto privilegiado ou destacado e a orientação para uma utilidade potencial do conhecimento produzido nos processos de decisão nos sistemas e serviços.

As definições para pesquisa em serviços de saúde encontradas na literatura deixam claros os contornos amplos e limites difusos dessa área temática:

- “A pesquisa em serviços de saúde pode ser definida simplesmente como o estudo científico das tarefas, recursos, atividades e resultados da prática clínica e dos serviços de saúde. Como a definição implica, são analisados muitos aspectos da atenção e, para tanto, engloba uma diversidade de disciplinas. Correndo o risco da simplificação excessiva, a pesquisa em serviços de saúde investiga três dimensões básicas, porém articuladas, da atenção: (1) os processos de decisão no cuidado realizado; (2) os processos envolvidos na oferta da atenção melhor possível e (3) os resultados da atenção" 24 (p. 99).

- "A pesquisa em serviços de saúde se interessa pela qualidade, efetividade e custo-efetividade da atenção à saúde, bem como pelo acesso à atenção à saúde para toda a população americana e pelos aperfeiçoamentos na operação do complexo sistema de saúde nacional" (AHRQ http://www.ahrq.gov/child/edlifesc.htm, acessado em 11/Mar/2004).

- “As pesquisas em serviços de saúde tipicamente se ocupam com questões relativas à orga- nização, financiamento, utilização e custos da atenção à saúde. Aumentar não só o acesso a uma atenção eficiente e de qualidade, como também a oferta desta, contribuirá, espera-se, para a saúde da população” 25 (p. 1641).

- “A pesquisa em serviços de saúde tem por objetivo produzir informações confiáveis e válidas que permitem o embasamento do desenvolvimento de serviços de saúde adequados, efetivos, custo-efetivos, eficientes e aceitáveis. Não é uma disciplina científica mas uma área de pesquisa aplicada que se apóia em um leque de disciplinas e perspectivas que incluem a antropologia, economia, epidemiologia, história, medicina, saúde pública, enfermagem, ciência política, ciências sociais, gestão e administração, avaliação tecnológica, estatística etc. Diferentemente da pesquisa clínica que enfoca o paciente e suas relações com o tratamento e cuidado em uma perspectiva individual, a pesquisa em serviços de saúde adota uma perspectiva populacional. As questões abordadas pelas pesquisas em serviços de saúde incluem: (a) Que tipo de atenção à saúde deve ser implantada/oferecida?; (b) Como os serviços podem e devem ser financiados?; (c) Quem pode e deve ser atendido em que serviços e como?; (d) Qual a qualidade da atenção realizada?" 26 (p. 156).

Ainda que muitas disciplinas possam contribuir para a produção de conhecimentos capazes de responder às questões formuladas nessa área temática, observa-se na literatura um predomínio de estudos referenciados pela medicina, enfermagem, epidemiologia, avaliação tecnológica e econômica, saúde pública, gestão e administração e ciências sociais e política. Quanto à metodologia adotada nesses estudos, observa-se também uma pluralidade de abordagens, com predomínio daquelas de natureza quantitativa, epidemiológicas e estatísticas, além de uma utilização crescente das metodologias de natureza qualitativa.

Uma característica importante das pesquisas em serviços de saúde tem sido a valorização de bancos de dados secundários, administrativos e demográficos/epidemiológicos, de documentos e da revisão da produção científica existente, como reflexo de uma preocupação com a sua viabilidade e reprodutibilidade. Em uma série de quatro artigos dedicados à metodologia para a pesquisa em serviços de saúde publicada no British Medical Journal, e parte de um livro publicado pela BMJ Publishing Group sobre o tema em 1998, foram abordadas as seguintes questões: (a) interpretando as evidências: a escolha entre estudos randomizados e não randomizados; (b) avaliação de intervenções na saúde por área ou por serviços; (c) in- 
trodução a métodos "bayesianos” na avaliação tecnológica em saúde; (d) lidando com a incerteza nas avaliações econômicas de intervenções na saúde $27,28,29,30$. Tais questões deixam evidente a pluralidade de abordagens e reconhecimento da necessidade de otimizar o conhecimento e os dados disponíveis:

Os artigos que se enquadram como pesquisa em serviços de saúde são publicados em um grande número de revistas da área da saúde, com perfil diversificado. Analisando o universo das revistas científicas de língua inglesa, observa-se que algumas delas têm essa área temática como uma questão central, por exemplo, a Health Services Research, ou dominante, como a Medical Care, Medical Care Research and Review e Nursing Outlook. Nas revistas da área de epidemiologia, refletindo as suas vocações e representações institucionais/profissionais diferenciadas, observa-se desde uma quase ausência do tema (American Journal of Epidemiology, Epidemiology), até seções regulares sobre a temática (International Journal of Epidemiology, European Journal of Epidemiology, Journal of Clinical Epidemiology). Também as revistas de saúde pública/medicina preventiva/ medicina social apresentam uma presença muito diferenciada da temática, destacando-se a sua forte presença nas revistas Social Science and Medicine, Health Policy e Health \& Place.

Além dessas, as revistas dedicadas às diferentes áreas de avaliação em saúde também publicam, evidentemente, com regularidade e em proporção elevada, artigos com um enfoque em pesquisa em serviços de saúde, particularmente aquelas da área de avaliação tecnológica em saúde (International Journal of Technology Assessment in Health Care), avaliação econômica em saúde (European Journal of Health Economics), avaliação de qualidade (International Journal for Quality in Health Care) e de programas (Journal of Evaluation in Clinical Practice). Convém destacar adicionalmente que, em termos do impacto dos artigos publicados, as revistas médicas "generalistas" tradicionais, como British Medical Journal, Lancet e Journal of the American Medical Association são importantes veículos de difusão para a produção na área temática. Finalmente, encontram-se artigos sobre essa temática em revistas vinculadas às especialidades médicas, com maior intensidade nas de pediatria, psiquiatria, cardiologia, obstetrícia e ginecologia e medicina intensiva. Não foram incluídos, nessa análise, os estudos desenvolvidos pelas agências nacionais européias, americanas e canadenses de avaliação tecnológica e econômica em saúde, publicados como relatórios técnicos, que se constituem, na sua maioria, em "pesquisa em serviços de saúde”, sendo acessíveis nos sites das agências.

Tomando como universo para análise apenas as revistas às quais se tem acesso pelo portal Periódicos/CAPES (http://www.periodicos. capes.gov.br), comprova-se que essa é uma área temática que transita por muitas especialidades, uma vez que todas as áreas do conhecimento que tratam da saúde e da atenção à saúde têm envolvimento com os sistemas e serviços de saúde. Por outro lado, essa condição dificulta a identificação de características que permitam agrupar essa produção em uma área temática reconhecida de forma ampliada (incluindo-se as agências de financiamento, programas de pós-graduação, comissões organizadoras de congressos etc.), ainda que assumidamente com limites bastante difusos. Essa dispersão também se comprova quando da realização de revisões bibliográficas, em que há necessidade da utilização de um número elevado de diferentes "unitermos" para a identificação dos artigos pertinentes à temática.

Reconhecendo de antemão a impossibilidade de uma categorização de subtemas que seja capaz de incluir de forma adequada e satisfatória todos os artigos publicados considerados inseridos na temática, propomos, para o aprofundamento da discussão, os seguintes subtemas principais, com exemplos de artigos recentes publicados nas revistas supracitadas:

- Processos de implantação de programas em serviços de saúde e estudos destes quanto às dimensões de estrutura e processos assistenciais $e$ organizacionais.

(1) Senior et al. 31: utilizando sistemas de informação existentes, analisaram-se as prescrições para depressão (antidepressivos), asma (broncodilatadores e corticóide inalatório) e diabetes (hipoglicemiantes e insulina) feitas por médicos generalistas de 131 clínicas no País de Gales, em 1997. Foram avaliadas variáveis relativas à necessidade (características sócio-econômicas, demográficas e epidemiológicas da clientela atendida) e relativas à oferta (tipo de clínica, perfil dos médicos, proximidade com hospital e especialistas). As análises revelaram que a necessidade exerce uma influência consistente sobre o volume e custos dos medicamentos prescritos, assim como as características da oferta determinam variações nos padrões das prescrições, identificando-se a existência de um cluster effect sobre o comportamento dos médicos, os quais se influenciam reciprocamente e são influenciados pelo contato com especialistas. Os autores consideram que estudos como esse podem contribuir para o planejamento do consumo de medicamentos. 
(2) Fitzpatrick et al. 32: inquérito realizado com pacientes submetidos à cirurgia para implantação de prótese de quadril em cinco regiões de saúde do Reino Unido. Foi aplicado questionário para classificação de gravidade da doença, informações quanto ao perfil sócio-econômico e demográfico, de tempo de espera para consulta com especialista, indicação e realização da cirurgia. Registraram-se longos períodos de espera, e observaram-se diferenças significativas para variáveis sociais, geográficas e de características do sistema de saúde. Os pacientes com mais dor e incapacidade na cirurgia revelaram intervalos de espera mais prolongados, indicando piora da condição clínica no período.

(3) La Veist et al. 33: utilizando base de dados desenvolvida para o estudo de pacientes com problemas cardiológicos atendidos em três hospitais da região de Baltimore, Estados Unidos, os autores propuseram três hipóteses para o estudo de diferenças raciais na utilização da angiografia coronariana: (a) pacientes que consultam cardiologistas são mais provavelmente encaminhados para angiografia coronariana; (b) pacientes afro-americanos têm menor probabilidade (em condições clínicas semelhantes) de ter consulta com cardiologista; (c) dos pacientes encaminhados para angiografia coronariana, não há diferenças raciais entre aqueles que a realizam. As análises segundo modelos multivariados e regressão logística confirmaram as hipóteses.

(4) Ham et al. 34: foram desenvolvidas análises comparativas de utilização de leitos hospitalares, valendo-se de bases de dados administrativos, para 2000 e 2001, em três sistemas de saúde com características diversas. Estudaram-se as hospitalizações, tempo de internação e dias / leito para a população acima de 65 anos de idade, com diagnóstico de entrada para 11 doenças principais. A comparabilidade dos diagnósticos foi garantida e foi feita a padronização por idade das três populações estudadas. O número de leitos/dia no sistema nacional de saúde britânico para os diagnósticos estudados é três vezes maior que o da Kaiser Permanente, um sistema de autogestão privado, e o dobro do número do Medicare. O estudo foi complementado com entrevistas a gestores dos sistemas e permitiu identificar diferenças na população atendida, assim como diferenças na gestão dos recursos assistenciais ambulatoriais e hospitalares.

- Impactos sobre a saúde da clientela ou população em geral das tecnologias de produto e/ou de processo inseridas nos processos de atenção dos serviços de saúde.
(5) Marrugat et al. 35: foram estudadas todos as pessoas de uma região da Espanha que receberam o diagnóstico de infarto agudo de miocárdio em 1996-1997. Foram utilizados dados de registro hospitalar de doenças cardiovasculares e de atestados de óbito com esse diagnóstico e adotaram-se critérios explícitos para classificação da condição clínica. Identificou-se uma letalidade mais elevada para pacientes acima de 65 anos, em condições clínicas comparáveis, menor proporção de encaminhamento para UTI e padrão de utilização diferenciado de procedimentos diagnósticos e terapêuticos, quando comparados com pacientes na faixa etária de 35-64 anos.

(6) Asplund et al. 36: foram usados os dados coletados para um estudo multicêntrico de drogas neuroprotetoras em pacientes com acidente vascular cerebral, incluindo-se nesse estudo pacientes de 19 países agrupados em dez regiões. Analisaram-se dados relativos a características clínicas e demográficas na admissão ao hospital, à utilização de recursos diagnósticos e terapêuticos e à sobrevida e condição clínica após três meses. Os resultados obtidos indicam diferenças estatisticamente diferentes entre as regiões analisadas para letalidade e condição clínica após três meses, as quais não puderam ser explicadas por ajustes no case-mix das amostras analisadas, nem pelo consumo de serviços na fase hospitalar ou após. Os autores sugerem que, possivelmente, existam diferenças clínicas não controladas e diferenças culturais importantes na abordagem dos casos de AVC, tanto pelos serviços de saúde, quanto pelas famílias, o que estaria influenciando as diferenças observadas.

(7) Banz et al. 37: avaliação dos riscos à saúde causados pela varicela e seu impacto econômico na Alemanha, com ênfase na relevância das complicações no tratamento como um componente importante de custo. Foi construído um modelo da epidemiologia da doença no país, sendo identificados e estimados os custos da atenção recebida nos serviços de saúde. Como base das estimativas, utilizaram-se as informações obtidas na vigilância epidemiológica dos casos de varicela. A análise do impacto econômico foi feita na perspectiva tanto da sociedade, quanto dos financiadores, apontando para custos diretos e indiretos significativos. Os autores consideram que a introdução da vacinação de rotina para varicela em base populacional teria uma relação custo-efetividade favorável.

- Políticas sociais e de saúde e suas relações com o desempenho dos sistemas de serviços de saúde. 
(8) Exworthy et al. 38: tomando como base documentos oficiais, análises secundárias e entrevistas com gestores de políticas públicas, foram identificadas as propostas políticas desenvolvidas no Reino Unido a partir da publicação do Acheson Report (1998), com objetivo de reduzir as iniqüidades em saúde, analisando-se seu impacto. Identificaram-se diversas iniciativas na área educacional, de política redistributiva e de atenção à saúde (priorizando grupos populacionais com risco aumentado para câncer, doenças cardiovasculares, acidentes e doença mental), voltadas para a atuação em fatores determinantes da iniqüidade. No entanto, observa-se pouco impacto sobre os indicadores de referência, e os autores utilizam o modelo das "janelas políticas" (policy windows) para explicar o impacto limitado das políticas desenvolvidas.

(9) Reeves \& Baker 39: para se obter maior impacto na atenção primária, há necessidade da sua articulação com programas de intervenção para grupos sociais vulneráveis. Foram construídas áreas geográficas visando à comparação das características sócio-econômicas e demográficas (percentual de idosos), e à cobertura de Programas de Atenção Primária e Programas Sociais. Observou-se que em áreas mais desfavorecidas socialmente havia menor cobertura da atenção primária e maior presença de programas sociais, e nas áreas com maior proporção de idosos essa relação era inversa, observando-se padrões complexos de articulação entre necessidades de atenção à saúde e necessidades de apoio social e a presença de programas voltados para o atendimento dessas necessidades.

Os exemplos selecionados confirmam a grande variabilidade nas abordagens e estratégias metodológicas adotadas, bem como a dificuldade no estabelecimento de classificações de subtemas com limites definidos. Observa-se a valorização do uso de informações administrativas e epidemiológicas existentes para o desenvolvimento de estudos que possam ser replicados e que sejam capazes de produzir conhecimentos que dialogam mais proximamente com as áreas executivas e operacionais. Podem também ser identificados alguns dos desafios colocados para a realização de pesquisas em serviços de saúde:

- Questões relativas à organização e operacionalização das pesquisas: Despertam nos profissionais e serviços estudados sentimentos de invasão e ameaça; necessitam de equipe de pesquisa multidisciplinar e estão freqüentemente condicionados por conjunturas e horizontes temporais.
- Questões metodológicas: A randomização e obtenção de controles adequados é difícil; presença do efeito "Hawthorne", isto é, a pesquisa introduz mudanças nas práticas; a escolha e a disponibilidade de variáveis de processo e resultado pertinentes e consistentes são cruciais; a utilização do conhecimento produzido nas pesquisas para conclusões mais ampliadas deve ser cuidadosa 24 .

Para identificar a presença de artigos com enfoques que possam ser considerados como de "pesquisa em serviços de saúde" na literatura nacional, e em particular na de saúde pública, foram realizados alguns procedimentos de busca e identificação de artigos: análise das duas principais revistas de saúde pública para o ano 2003 e busca com os unitermos "pesquisa em serviços de saúde”, "utilização de serviços de saúde" e "hospital", na base SciELO (Scientific Electronic Library Online - http://www. scielo.org). Nas duas revistas, a presença de artigos nessa temática é pouco freqüente, abaixo da média de um artigo/mês. Os artigos em que há referência aos serviços de saúde geralmente apresentam-se como uma variável em estudos epidemiológicos populacionais, não havendo, porém, uma caracterização dos mesmos que permita apreender a sua participação, na qualidade de portador de práticas assistenciais específicas, na epidemiologia do problema de saúde estudado.

Para o unitermo "health services research", sem limitação do período, na base SciELO, foram identificados apenas 11 artigos, publicados quase totalmente nas revistas de saúde pública. A utilização dos unitermos "health services utilization" e "hospital", ainda na base SciELO e sem limitação de período, permitiu a recuperação de 45 artigos. Nestes, predominam ainda as revistas de saúde pública/saúde coletiva, mas aparecem também revistas da área de pediatria, obstetrícia, psiquiatria, neurologia e assistência hospitalar. Destaca-se o importante número de artigos, publicados nos últimos cinco anos, que utilizam a base de dados do SIH/SUS de atenção hospitalar como fonte de informação para as análises desenvolvidas, bem como artigos que utilizam bases de dados da Fundação Instituto Brasileiro de Geografia e Estatística, como a Pesquisa Nacional de Amostra Domiciliar (PNAD) e Assistência Médico-Sanitária (AMS). Nesse caso, há uma proximidade com os estudos internacionais, mas essa produção se mostra bastante concentrada em termos regionais e institucionais.

A utilização de unitermos relativos a "atenção primária”, "atenção básica”, "atenção ambulatorial", "exames laboratoriais" e "procedi- 
mentos diagnósticos e terapêuticos" como estratégia de busca na base SciELO recuperou um número mais restrito de artigos. Observa-se que, para esse nível da atenção, a abordagem predominante nos artigos se dá na perspectiva de programas específicos (pré-natal, saúde da família), em que ora se enfatizam dimensões de gestão e financiamento, ora se analisam alguns indicadores gerais de desempenho do programa em uma perspectiva populacional (por exemplo: número de consultas, cobertura), geralmente sem detalhamento das características dos serviços e da assistência realizada.

Em síntese, é possível considerar que a produção bibliográfica de artigos relativos à pesquisa em serviços de saúde, com o recorte adotado, nas revistas brasileiras é pequena, tanto em números absolutos quanto proporcionalmente (levando-se em conta o pequeno número de revistas disponíveis). Podem estar sendo publicados artigos de pesquisadores brasileiros sobre essa temática em revistas internacionais, mas é pouco provável que isso esteja acontecendo em grande escala, em face da perspectiva de se tratar da produção de um conhecimento "aplicado" (e aplicável).

Nos últimos anos tem havido indiscutivelmente um investimento em "epidemiologia em serviços de saúde” por parte dos gestores e profissionais envolvidos na organização e prestação da atenção à saúde e da vigilância epidemiológica e sanitária 40. Embora seja parte da atividade profissional e rotina de trabalho, com desenvolvimento de bases de dados e sistemas de monitoramento, essa atividade praticamente não tem logrado levar ao desenvolvimento de pesquisas específicas, elaboração de artigos e publicação em revistas científicas. Uma interface importante da pesquisa em serviços de saúde no Brasil se dá com a produção na área das políticas sociais e de saúde, bastante consolidada e com maior articulação com a implementação das diretrizes políticas no SUS.

A importância, necessidade, utilidade e pertinência da realização e disseminação dos resultados de pesquisas em serviços de saúde está evidente. Também está claro que é preciso aumentar a produção nessa área temática no Brasil, principalmente considerando-se o crescente interesse no desenvolvimento e utilização de indicadores para o monitoramento do desempenho do SUS e apoio nas decisões sobre a distribuição dos recursos financeiros para os municípios. Recentemente, concluiu-se um projeto multicêntrico nacional de Desenvolvimento de Metodologia de Avaliação do Desempenho do Sistema de Saúde Brasileiro (http://www.proadess.cict.fiocruz.br). O proje- to, coordenado pela Associação de Pós-graduação em Saúde Coletiva (ABRASCO) e financiado pela Financiadora de Estudos e Projetos (FINEP), propôs uma matriz de dimensões a serem consideradas na definição dos indicadores, incluindo-se entre as quais a acessibilidade, efetividade, eficiência, aceitabilidade dos serviços de saúde. Para que seja possível o desenvolvimento de indicadores com critérios e metas pertinentes ao contexto brasileiro, mostra-se essencial o desenvolvimento de pesquisas que possam orientar essas definições, como já tem sido possível constatar na implementação dos indicadores do Pacto de Atenção Básica (http://www.saude.sp.gov.br/indicadores).

A tradução e incorporação dos resultados das pesquisas na prática dos profissionais e dos gerentes e gestores não ocorrem automática ou naturalmente 41. Alguns países (Reino Unido, Estados Unidos, Canadá) implementaram regulamentos e processos administrativos vinculando diretamente a necessidade da demonstração de uma "evidência científica" às decisões de financiamento, aprovação de demandas de incorporação e utilização de tecnologias, ou estabelecimento de prioridades para a atenção e políticas de saúde. Contudo, os resultados observados foram bastante diversos do pretendido, apontando para a participação sempre decisiva de outros elementos nos processos de decisão 42,43,44.

Outras iniciativas, como a criação de bases de dados e de literatura científica orientadas para a produção de sínteses capazes de orientar as práticas profissionais, o desenvolvimento de pesquisas com temas e abordagens mais próximos às questões relativas à organização e qualidade da assistência, além da produção de conhecimentos capazes de orientar as iniciativas que visam a facilitar as mudanças nas práticas organizacionais e profissionais, não têm obtido o êxito pretendido, ainda que se observem lentas mudanças culturais e institucionais que indicam atitudes e práticas cotidianas mais receptivas à informação técnica e científica $45,46,47,48,49$.

Programas especialmente dirigidos para a otimização do conhecimento produzido e a sua utilização na rotina dos sistemas e serviços de saúde, como o Center for Outcomes and Effectiveness Research (COER) da Agency for Healthcare and Policy Research (AHCPR) e National Quality Measurement and Reporting System (NQMRS) para os hospitais americanos, também identificaram dificuldades maiores do que as esperadas no momento da sua formulação 50,51. O relatório final para o Outcomes Research refere que foi financiado um grande número de 
pesquisas nos seus nove anos de atividade e que os investigadores mostraram-se bem-sucedidos em produzir relatos progressivamente mais acurados e detalhados sobre a assistência à saúde desenvolvida, como também no desenvolvimento de instrumentos para medir custos e resultados da atenção. Todavia, os pesquisadores principais entrevistados consideram que poucas pesquisas influenciaram $\mathrm{mu}$ danças na gestão, nas decisões clínicas ou contribuíram para mudanças na qualidade e nos custos da assistência, destacando os AVCs como o único problema de saúde que parece ter sido objeto de mudanças nas condutas em conseqüência das pesquisas desenvolvidas.

Um dos principais desafios da nova geração de pesquisas voltadas para a produção de impactos positivos sobre os resultados da atenção e da saúde populacional será ter capacidade de ultrapassar as descrições e o aprimoramento metodológico e contribuir para a solução de problemas e melhoria da qualidade da assistência e da implementação efetiva de políticas intersetoriais 52 (http://www.ahrq.gov/clinic/ outcosum.htm, acessado em 11/Mar/2004). São apontadas na literatura como questões importantes de pesquisa e atuação o investimento em: desenvolvimento de indicadores de avaliação de desempenho e impacto em saúde, revisões de literatura sobre programas para a saúde da população e intervenções efetivas sobre esta, estudos de caso de intervenções intersetoriais, desenvolvimento de bancos de dados populacionais longitudinais e de acesso e utilização de serviços de saúde 21 .

Em artigo publicado no British Medical Journal 53, que discute as alternativas para aumentar o impacto das pesquisas em serviços de saú- de, os autores apontam as dificuldades identificadas pelos envolvidos nesses estudos no Reino Unido:

- Pesquisadores: frustram-se pela baixa utilização dos resultados das suas pesquisas pelos gestores e gerentes e pela pouca valorização científica do conhecimento produzido.

- Gerentes/administradores: não identificam informações relevantes nas pesquisas desenvolvidas e consideram que seu financiamento é um desperdício de recursos.

- Gestores: preocupam-se com a temporalidade das pesquisas - para serem úteis precisam estar disponíveis nos momentos de decisão.

- Todos os grupos identificam a necessidade urgente de articular de forma efetiva aqueles que financiam, utilizam e produzem as pesquisas em serviços de saúde.

Sem dúvida, a identificação das potencialidades e das limitações da pesquisa em sistemas e serviços de saúde, além do desenvolvimento de fluxos de comunicação oportunos e compartilhados entre os interessados, poderá contribuir para uma otimização dos esforços, e, neste texto, foi possível identificar uma progressiva maior visibilidade dessa temática. Portanto, parece indiscutível que o desenvolvimento de pesquisas em sistemas e serviços de saúde poderá contribuir para o aprimoramento e fortalecimento do Sistema Único de Saúde e para a qualidade na atenção nos serviços de saúde, integrando-se aos processos sociais, econômicos e políticos que constroem a saúde atual e futura da população brasileira, fortalecendo da melhor forma possível o papel que a ciência e a tecnologia podem e devem ter nas sociedades contemporâneas 12,54.

\section{Resumo}

Os serviços e sistemas de saúde têm uma participação importante na determinação dos níveis de saúde e condições de vida das populações atualmente. Aprofundar e ampliar o conhecimento sobre eles, na perspectiva da acessibilidade, desempenho e impacto dos sistemas, e sobre as condições e qualidade da atenção prestada em contextos específicos, podendo se constituir em apoio às decisões de gestores, gerentes e profissionais da área da saúde, é o objetivo de uma área temática multidisciplinar de "pesquisa em serviços de saúde". Neste texto, através da análise da literatura internacional recente, discutem-se as principais vertentes e características dessa produção, bem como algumas das questões que essa análise coloca para a pesquisa nessa área temática no Brasil.

Serviços de Saúde; Pesquisa sobre Serviços de Saúde; Qualidade da Assistência à Saúde, Acesso e Avaliação 


\section{Referências}

1. Goddard M, Smith P. Equity of access to health care services: theory and evidence from the UK. Soc Sci Med 2001; 53:1149-62.

2. Silva JA, Dalmaso ASW. Agente comunitário de saúde: o ser, o saber, o fazer. Rio de Janeiro: Editora Fiocruz; 2002.

3. White KL. Investigaciones sobre servicios de salud: uma antología. Washington DC: Organización Panamericana de la Salud; 1992.

4. Hill GB. Archie Cochrane and his legacy. An internal challenge to physicians "autonomy"? J Clin Epidemiol 2000; 53:1189-92.

5. White KL. Jerry Morris and health services research in the USA. Int J Epidemiol 2002; 31:690-2.

6. Novaes HMD. Epidemiologia e avaliação em serviços de atenção médica: novas tendências na pesquisa. Cad Saúde Pública 1996; 12 Suppl 2:7-12.

7. Furtado J. A indústria de equipamentos médicohospitalares: elementos para uma caracterização da sua dimensão internacional. In: Negri B, Giovanni $\mathrm{G}$, organizadores. Brasil: radiografia da saúde. Campinas: Editora Unicamp; 2001. p. 45-62.

8. Queiroz S, Gonzáles A. Mudanças recentes na estrutura produtiva da indústria farmacêutica. In: Negri B, Giovanni G, organizadores. Brasil: radiografia da saúde. Campinas: Editora Unicamp; 2001. p. 123-56.

9. McDaid D, Cookson R. Evaluating health care interventions in the European Union. Health Policy 2003; 63:133-9.

10. Maynard A, McDaid D. Evaluating health interventions: exploring the potential. Health Policy 2003; 63:215-26.

11. Novaes HMD, Goldbaum M, Carvalheiro JR. Políticas científicas e tecnológicas e saúde. Revista USP 2001; 51:28-37.

12. Ginzberg E. Health services research. Key to health policy. Cambridge: Harvard University Press; 1991.

13. Cohn A. Estado e sociedade e as reconfigurações do direito à saúde. Ciênc Saúde Coletiva 2003; 8:919.

14. Raymond S. Science, technology and the economic future. New York: New York Academy of Sciences; 1998.

15. Almeida CM. Reforma do Estado e reforma de sistemas de saúde: experiências internacionais e tendências de mudança. Ciênc Saúde Coletiva 1999; 4:263-86.

16. Lécourt D. Contre la peur. Paris: Hachette; 1990.

17. Stengers I. L'invention des sciences modernes. Paris: La Découverte; 1993.

18. Rancière J. "O Dissenso". In: Novaes A, organizador. A crise da razão. São Paulo: Cia. das Letras/ Brasília: Ministério da Cultura/Rio de Janeiro: Fundação Nacional de Arte; 1996. p. 367-82.

19. Greer AL, Freeman JL, Goodwin JS, Wu ZH. Bringing the patient back in: guidelines, practice variations, and the social context of medical practice. Int J Technol Assess Health Care 2002; 18:747-61.

20. McGlynn EA. Introduction and overview of the conceptual framework for a national quality measurement and reporting system. Med Care 2003; 42 Suppl:I1-7.

21. Kindig D, Day P, Fox DM, Gibson M, Knickman J,
Lomas J, et al. What new knowledge would help policymakers better balance investments for optimal health outcomes? Health Serv Res 2003; 38:1923-37.

22. McCarthy M. Public health research in Europe. Lancet 2002; 360:1439.

23. Leatherman S, Hibbard JH, McGlynn E. A research agenda to advance quality measurement and improvement. Med Care 2003; 41:I80-6.

24. Scott I, Campbell D. Health services research: what is it and what does it offer? Intern Med J 2002; 32:91-9.

25. Lurie N, McLaughlin C, House JS. Guest editors' introduction: in pursuit of the social determinants of health: the evolution of health services research. Health Serv Res 2003; 38:1641-3.

26. Fulop N, Allen P, Clarke A, Black N. From health technology assessment to research on the organization and delivery of health services: addressing the balance. Health Policy 2003; 63:155-65.

27. McKee M, Britton A, Black N, McPherson K, Sanderson C, Bain C. Interpreting the evidence: choosing between randomized and non-randomised studies. BMJ 1999; 319:312-5.

28. Ukoumunne OC, Gulliford MC, Chinn S, Sterne JAC, Burney PGJ, Donner A. Evaluation of health interventions at area and organization level. BMJ 1999; 319:376-9.

29. Spiegelhalter DJ, Myles JP, Jones DR, Abrams KR. An introduction to Bayesian methods in health technology assessment. BMJ 1999; 319:508-12.

30. Briggs AH, Gray AM. Handling uncertainty in economic evaluations of healthcare interventions. BMJ 1999; 319:635-8.

31. Senior ML, Williams H, Higgs G. Morbidity, deprivation and drug prescribing: factors affecting variations in prescribing between doctors' practices. Health Place 2003; 9:281-9.

32. Fitzpatrick R, Norquist JM, Reeves BC, Morris RW, Murray DW, Gregg PJ. Equity and need when waiting for total hip replacement surgery. J Eval Clin Pract 2004; 10:3-9.

33. LaVeist TA, Arthur M, Morgan A, Plantholt S, Rubinstein M. Explaining racial differences in receipt of coronary angiography: the role of physician referral and physician specialty. Med Care Res Rev 2003; 4:453-67.

34. Ham C, York N, Sutch S, Shaw R. Hospital bed utilization in the NHS, Kaiser Permanente, and the US Medicare programme: analysis of routine data. BMJ 2003; 327:1257-60.

35. Marrugat J, Sala J, Manresa JM, Gil M, Elosua R, Perez G, et al. Acute myocardial infarction population incidence and in-hospital management factors associated to 28-day case-fatality in the 65 year and older. Eur J Epidemiol 2004; 19:231-7.

36. Asplund K, Ashburner S, Cargill K, Hux M, Lees K, Drummond M. Health stroke resource use and stroke outcome. Multinational comparisons within the GAIN international trial. Int J Technol Assess Health Care 2003; 19:276-7.

37. Banz K, Wagenpfeil S, Neiss A, Hammerschmidt T, Wutzler P. The burden of varicella in Germany. Eur J Health Econo 2004; 5:46-53. 
38. Exworthy M, Blane D, Marmot M. Tackling health inequalities in the United Kingdom: the progress and pitfalls of policy. Health Serv Res 2003; 38 (6 Part II):1905-21.

39. Reeves D, Baker D. Investigating relationships between health need, primary care and social care using routine statistics. Health Place 2004; 10:12940.

40. Barreto ML. Papel da epidemiologia no desenvolvimento do Sistema Único de Saúde no Brasil: histórico, fundamentos e perspectivas. Rev Bras Epidemiol 2002; 5 Suppl:4-17.

41. Gonzales JJ, Ringeisen HL, Chambers DA. The tangled and thorny path of science to practice: tensions in interpreting and applying "evidence". Clinical Psychology: Science and Practice 2002; 9:204-9.

42. Claxton K, Sculpher M, Drummond M. A rational framework for decision making by the National Institute for Clinical Excellence (NICE). Lancet 2002; 360:711-5.

43. Oberlander J, Marmor T, Jacobs L. Rationing medical care: rhetoric and reality in the Oregon Health Plan. CMAJ 2001; 164:1583-7.

44. Mackenbach JP, Bakker M. Tackling socioeconomic inequalities in health: analysis of European experiences. Lancet 2003; 362:1409-14.

45. Glasziou P, Vandenbroucke J, Chalmers I. Assessing the quality of research. BMJ 2001; 328:39-41.
46. McCarthy M. Researchers try marketing techniques to sell their results. Lancet 2003; 362:1204-5.

47. Grol R, Grimshaw J. From best evidence to best practice: effective implementation of change in patients' care. Lancet 2003; 362:1225-30.

48. De Maeseneer JM, van Driel ML, van Weel C. The need for research in primary care. Lancet 2003; 363:1314-9.

49. Lanier DC, Roland M, Burstin H, Knottnerus JA. Doctor performance and public accountability. Lancet 2003; 362:1404-8.

50. Berwick DM, Brent J, Coye MJ. Connections between quality measurement and improvement. Med Care 2003; 41:I30-8.

51. Jefford M, Stockler MR, Tattersall MHN. Outcomes research: what is it and why does it matter? Intern Med J 2003; 33:110-3.

52. Lomas J. Health services research. BMJ 2003; 327: 1301-2.

53. Dash P, Gowman N, Traynor M. Increasing the impact of health services research. BMJ 2003; 327: 1339-41.

54. Brown LD. Knowledge and power: health services research as a political resource. In: Ginzberg E, editor. Health services research. Cambridge: Harvard University Press; 1991. p. 20-45.

Recebido em 27/Abr/2004

Aprovado em 10/Mai/2004 